\title{
Influence of the Fabrication Process on the Performance of $X$-Band Filters
}

\author{
W. M. Ali, C. Min, C. E. Free \\ Advanced Technology Institute \\ University of Surrey, Guildford Surrey GU2 7XH, UK
}

\begin{abstract}
Growing demand for mobile and wireless communication systems has generated much interest in low-cost, high quality microwave fabrication technologies. The performance of these systems relies on not only excellent design and analysis but also the characteristics of materials and their corresponding fabrication technologies. Often the designer is faced with a compromise between satisfying the high performance demands of a microwave component, and achieving low fabrication cost. This paper studies feasibility of realizing microwave bandpass filters using thick film screen printing techniques. The measured and simulated results for a $9.5 \mathrm{GHz}$ edge coupled bandpass filter have been determined and compared.
\end{abstract}

Keywords: bandpass filter, thick film technology, fabrication error.

\section{INTRODUCTION}

In recent years, the improved characteristics of thick film technology make it highly applicable to modern commercial, industrial, and military applications. The advances in thick film technology, notably in terms of lower material losses and finer line definitions, have been enabled this relatively low-cost process to become a viable option for the development of microwave passive components. The factors of size reduction, low loss, mass production, and cost effectiveness are essential issues that need to be considered when seeking to achieve optimum component design. This paper addresses some of the key issues of performance and repeatability through investigations on a practical $X$-band thick-film filter.

Filters play an important role in modern wireless communication systems for out-of-band rejection to prevent inter-channel interference (ICI) and wide bandwidth requirements for system operation. In this work a $9.5 \mathrm{GHz}$ edge coupled bandpass filter in single layer configuration is fabricated on typical alumina substrate with a thickness of $635 \mu \mathrm{m}$ and relative permittivity $\left(\varepsilon_{r}\right)$ of about 9.8 , thus leads to reduction in component size. Care should be taken for microstrip line structure using screen printing process such that the characteristics of design are dominated by the dimensions and definitions of lines.

Owing to many uncertain factors during characterization process such as structural shrinkage after firing, error allowance of substrate production, and imperfect mesh screen printing, some analysis on the sources of error of physical dimensions are performed to have not only a prediction of circuit performance but also a reference for future design considerations. The RF performance is tested by measurements using a vector network analyzer and the measured results are compared with simulation.

\section{THICK FILM TECHNOLOGY}

Thick film technology is a highly flexible and low-cost fabrication process for many applications such as digital integrated circuits, high-power and high-voltage circuits, and also high frequency microwave circuits. The thick films are produced by screen printing of conducting and insulating materials on ceramic substrates [1].

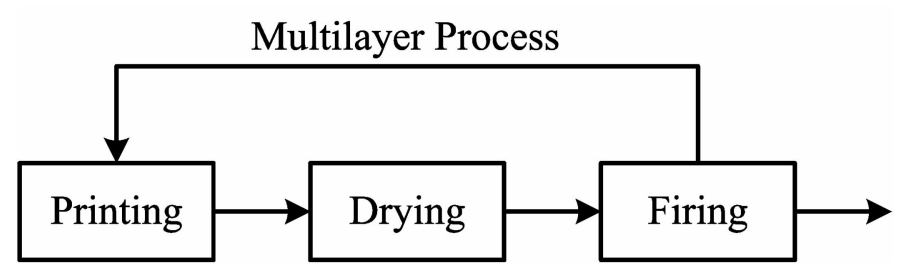

Figure 1. Thick Film Process

Fig. 1 shows the thick film process that was used to fabricate the bandpass filter in this study. The standard screenprinting process includes the three conventional steps of pattern printing, drying, and firing. Printed-line definition is dominated by the mesh density which includes the diameter of mesh wire for the shapes of lines. The paste viscosity is influenced by room temperature, the pressure, angular position and hardness of squeegee are all significant factors in establishing the thickness of the print. The thick film paste is applied as a pattern by screen printing over the total area of the insulating substrate. Drying process is applied on the printed thick film layer to remove volatile solvent. And it is performed in a natural-drying oven with an optimized temperature of $80^{\circ} \mathrm{C}$ for 20 minutes. The firing is performed in programmable chamber furnace to provide the high density thick film structure. The firing is made at temperature around $850^{\circ} \mathrm{C}$ for 6 to 8 hours to complete the entire firing process. The firing process not only removes the remaining organic binders but also develops the electrical properties [2]. When the operating frequency of design is higher, higher modes become excited. Therefore, thinner substrates are required to avoid the higher order modes influencing the performance of the circuits. The surface roughness of the substrate is important because it will affect the surface roughness on the bottom of the printed conductor layer. Surface smoothness of the printed layer relies on the stroke 
stability of the squeegee and the fluidity of the blade. Therefore, optimized settings of the printing machine including down-force pressure, and speed of stroke movement, are required. The screen is composed of an emulsion layer and stainless steel mesh with an assembling frame for robustness and stability during the printing action. The recommended emulsion thickness of their corresponding mesh counts for conductor paste printing is 18 um (325 Mesh). These settings produce the fired thickness of conductor to be about 10-12um per layer [3].

\section{FILTER DESIGN}

In general, the edge coupled microstrip bandpass filters, with bandwidths less than about $20 \%$ can be easily fabricated. But, very tightly coupled lines are needed if wider bandwidth filters are required. By reducing the substrate thickness, the track separation becomes smaller. However, this small track separation can be a problem during the circuit fabrication [4]. The trade-off between substrate thickness, minimum coupling gap, characteristics impedance and overall loss is a very important issue that needs to be considered to achieve the required filter performance.

In this study, several samples of 7-section bandpass filter have been designed with a center frequency of $9.5 \mathrm{GHz}$ with required fractional bandwidth of $10 \%$, and a roll-off specified by $|\mathrm{S} 21|<-30 \mathrm{~dB}$ at $8 \mathrm{GHz}$ and $10 \mathrm{GHz}$. The filters were fabricated on Alumina substrate $\left(\varepsilon_{r}=9.8\right)$ with a thickness of $635 \mu \mathrm{m}$. And the physical dimensions of each coupled section are shown in Table I.

TABLE I

The SimUlated DimENSIONS OF THE BANDPASS FILTER AT $9.423 \mathrm{GHz}$

\begin{tabular}{|c|c|c|c|c|c|c|c|}
\hline Section & $\mathbf{1}$ & $\mathbf{2}$ & $\mathbf{3}$ & $\mathbf{4}$ & $\mathbf{5}$ & $\mathbf{6}$ & $\mathbf{7}$ \\
\hline Width $(W)$ & 0.413 & 0.635 & 0.635 & 0.635 & 0.635 & 0.635 & 0.413 \\
\hline Space $(S)$ & 0.175 & 0.568 & 0.873 & 0.873 & 0.873 & 0.568 & 0.175 \\
\hline Length $(L)$ & 3.053 & 2.945 & 2.929 & 2.929 & 2.929 & 2.945 & 3.053 \\
\hline \multicolumn{10}{|c}{} \\
\hline
\end{tabular}

The physical dimensions were inserted into a full-wave simulator for verification of the circuit performance. Fig.2 and Fig.3 show the simulated and measured responses of the design. The filter design has a center frequency of $9.423 \mathrm{GHz}$ with $3 \mathrm{~dB}$ bandwidth which was about $1.06 \mathrm{GHz}(11.3 \%)$ and roll-off of $|\mathrm{S} 21|<-30 \mathrm{~dB}$ at $8 \mathrm{GHz}$ and $10.4 \mathrm{GHz}$. The measured circuit has a center frequency of $9.527 \mathrm{GHz}$ with $3 \mathrm{~dB}$ bandwidth which about $1 \mathrm{GHz}(10.5 \%)$ and roll-off of $|\mathrm{S} 21|<-$ $30 \mathrm{~dB}$ at $8.62 \mathrm{GHz}$ and $10.39 \mathrm{GHz}$ with insertion loss of $1.84 \mathrm{~dB}$. It is seen from the results that the bandwidth slightly shrinks. The reason was mainly due to the shrinkages of the conductor layer, resulting in the increase of spacing of each filter section. And this may be also the reason for the shift of design's centre frequency in comparison with the specifications. Although the losses due to the dielectric material and bulk conductors had been included in the simulation, the additional insertion loss may be due to the surface roughness of the fired circuits. This effect becomes announced in mm-wave frequencies, and should be included in the design.

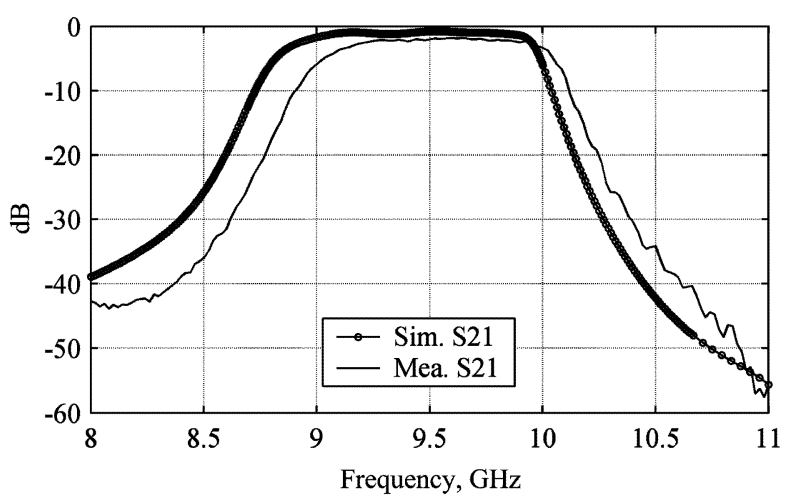

Figure 2. Simulated and measured insertion loss of the design.

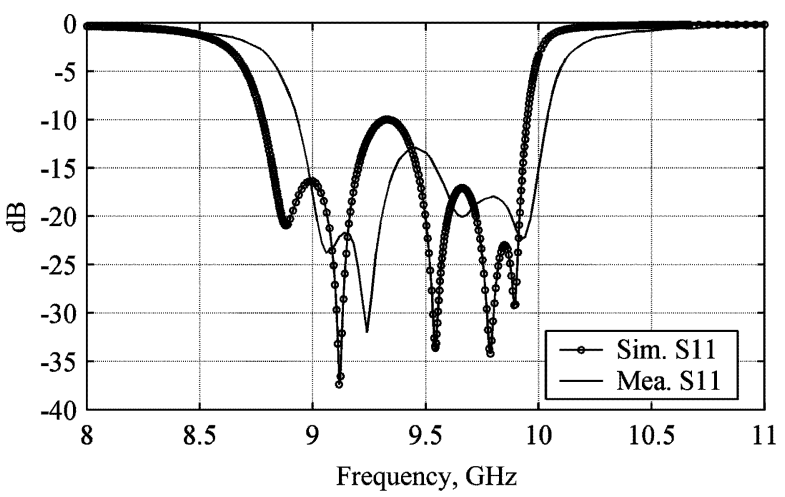

Figure 3. Simulated and measured return loss of the design.

The microstrip line structures are sensitive to their line definitions and dimensions. Changes of dimensions will alter their characteristics and have influence on component design of such structure. Since thick-film fabrication technology is being used there will be some shrinkage when the printed conductors are fired. Shrinkage of final fired circuits results in the changes of dimensions and performance. Analysis on the dimensions tolerance is performed to check any influences occurred after the firing process.

\section{FABRICATION ERROR ANALYSIS}

Due to the variation in circuit geometries that can occur during a thick-film processing cycle, some analysis of the effects of these errors is essential. A number circuit samples were fabricated by completing the same stages of the fabrication process under identical conditions using a semiautomatic screen printer.

Fig. 4 shows the comparison of the insertion loss with different changes of physical dimensions, whilst Fig. 5 is the variation of the impedance matching within pass band for the different cases. A five percent decrease of the filter's lengths and widths results in a narrower bandwidth of about $10.57 \%$ 
with worse roll-off, and a slight downward shift of the center frequency of about $2.71 \%$. A five percent of increase of dimensions results in a wider bandwidth of about $12.2 \%$ with better roll-off, and slightly upward shift of the center frequency of around $2.46 \%$. It is reasonable since decrease of line width results in larger spacing of each filter section and therefore narrower bandwidth. This analysis gives a useful insight into the effect on the filter's electrical performance, due to shrinkage occurring during the firing process.

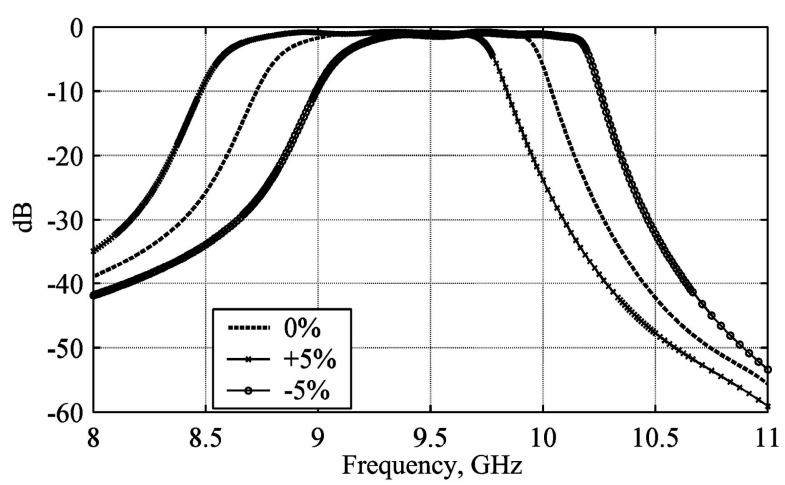

Figure 4. Filter insertion loss comparison for different fabrication error.

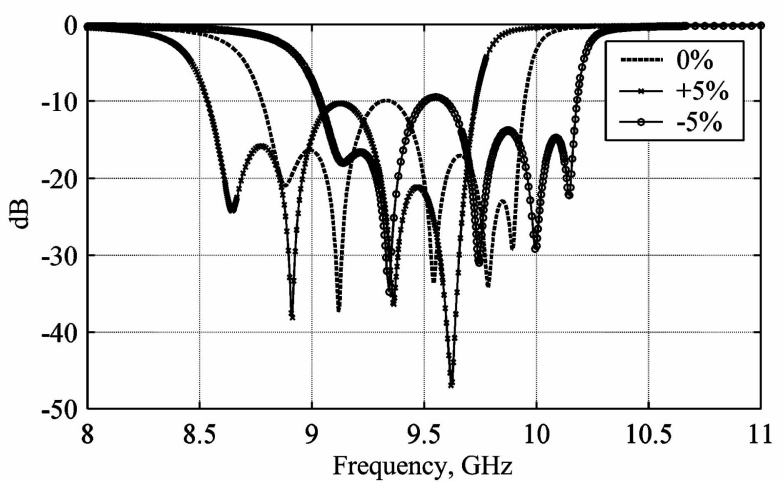

Figure 5. Simulated return loss results for different fabrication errors.

TABLE. II

Comparison between the simulated and measured results

\begin{tabular}{|c|c|c|c|c|}
\hline Sample & $\boldsymbol{f}_{\boldsymbol{0}}(\mathbf{G H z})$ & $\boldsymbol{B} \boldsymbol{W}(\mathbf{\%})$ & $\boldsymbol{I L} \mathbf{( d B )}$ & $\Delta \boldsymbol{f}_{\boldsymbol{o}}(\mathbf{\%})$ \\
\hline Simulation & 9.423 & 11.30 & 0.77 & - \\
\hline$\# 1$ & 9.530 & 10.60 & 1.68 & 1.14 \\
\hline$\# 2$ & 9.550 & 10.47 & 1.64 & 1.35 \\
\hline$\# 3$ & 9.540 & 10.80 & 1.49 & 1.24 \\
\hline$\# 4$ & 9.527 & 10.50 & 1.84 & 1.10 \\
\hline
\end{tabular}

Table II shows a comparison between the measured results for the circuit samples with the results from simulation. The measured insertion loss $(I L)$ level within pass band was about
$1.66 \mathrm{~dB}$ in average. The average center frequency $\left(f_{0}\right)$ of these samples was measured to be about $9.53 \mathrm{GHz}$ with $3 \mathrm{~dB}$ bandwidth $(B W)$ of $1 \mathrm{GHz}(10.59 \%)$. The slight decrease of bandwidth may be due to the shrinkage of the conductor which was found to be about $1 \%$, and is acceptably small. Sample \#3 can be regarded as the best one with the minimal insertion loss, widest bandwidth, and moderate shrinkage compared with the simulation results.

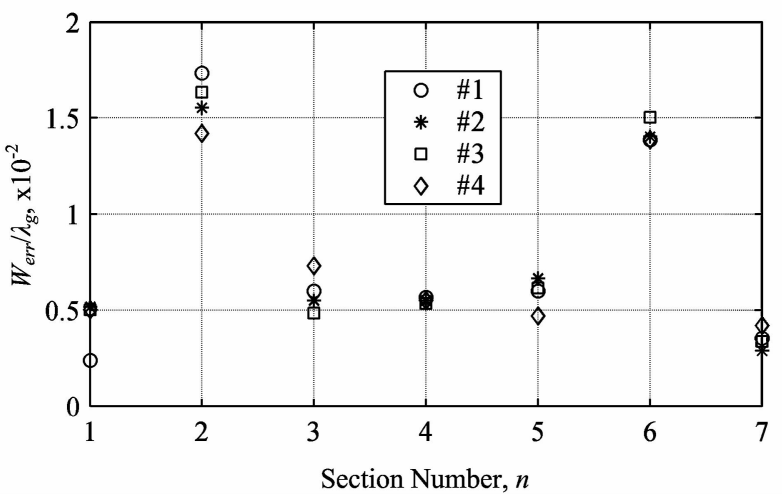

Figure 6. Measured width error versus section number.

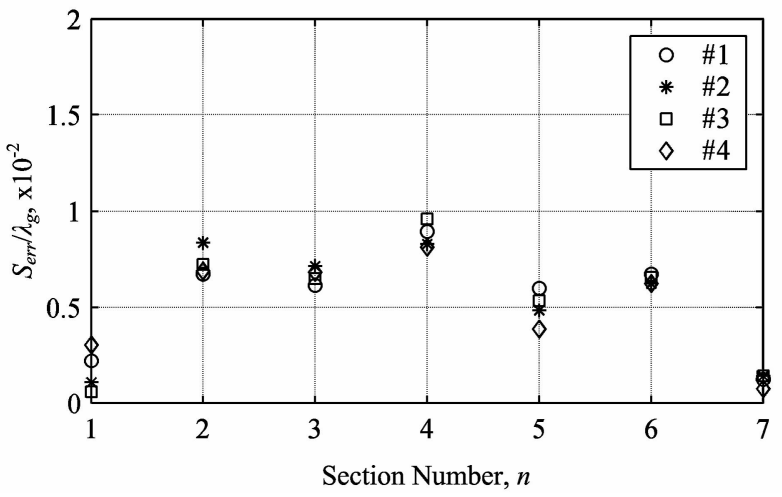

Figure 7. Measured spacing error versus section number.

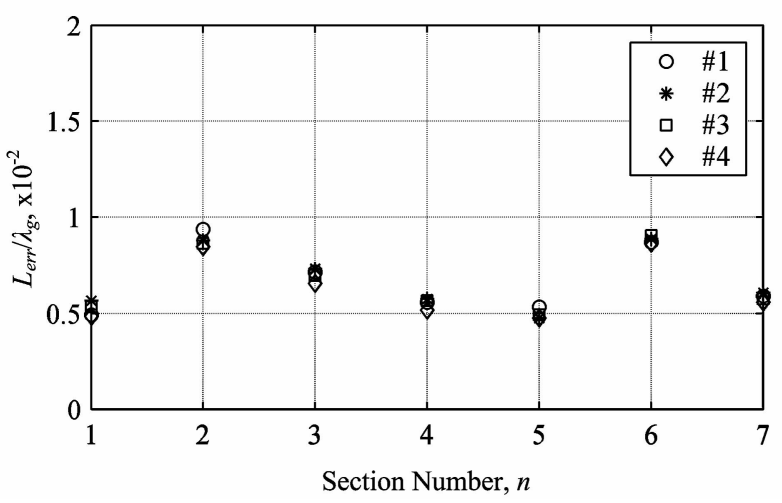

Figure 8. Measured length error versus section number of the samples. 
The variations of the dimensions of the filter elements are crucial to the performance of the component. In order to understand the effect of the fabrication process, it is essential to obtain accurate information about the variations in the widths and spacings of the conductors. These aspects will assume greater importance as the frequency of the design is extended into the millimetre-wave region. In the present study, microscopic examination of the fabricated circuits was used to obtain accurate dimensional data after firing.

The variations between the measured and designed dimensions namely, $W_{e r r}, S_{e r r}$ and $L_{\text {err }}$, were defined and measured relatively to the guide wavelength at the design's center frequency. It was found from Fig. 6 that the variations of widths are relatively small compared with the dispersive properties of the microstrip line structures. This is also evident from the measured passband return loss shown in Fig. 3. Fig. 7 shows the measured spacing error of each filter section versus section number, for the four samples. It is seen that the sample \#3 has the minimum $S_{e r r}$ at the first section, which dominates the design's operating bandwidth. Fig. 8 shows the measured lengths error ratio versus section number of the samples. It is found that sample \#4 has the smallest length error while sample \#2 has the biggest length error. These results matched with the results which were found from Table II such that the sample \#3 has the minimal $S_{e r r}$ and a measured bandwidth that is closest to the desired value. This is reasonable since the decrease in the spacing of each section results in an increase in bandwidth. In summary, from the measured results, the width error has influence on the impedance matching while any spacing error results in the variations of the design's operating bandwidth. The slight shift in the centre frequency may have been due to shrinkage effects, but more likely to incorrect compensation for electromagnetic fringing at the end of each section. Although the losses due to the dielectric material and bulk conductors had been included in the simulation, the additional insertion loss may be due to the surface roughness of the fired circuits. This measurement-based analysis forms not only predictable data but also applicable information for circuit designers, where the compensation of dimensions to account for fabrication errors are required at the design stage.

\section{CONCLUSION}

The work has shown that shrinkage effects during the fabrication of thick-film planar circuits can have a significant effect on the electrical performance. However, data for a series of components fabricated under near-identical processing conditions has shown the shrinkage effects are relatively predictable, and can be taken account of in the initial circuit design. Thus the results demonstrate the shrinkage that occurs in the processing of thick-film components does not detract from the low-cost, high-volume production advantages offered by thick-film technology.

\section{ACKNOWLEDGMENT}

The authors would like to thank Dr. M. Henry of the Advanced Technology Institute at the University of Surrey, for her help during the fabrication process of the filter circuit and they also would like to acknowledge the financial support from Kuwaiti Government.

\section{REFERENCES}

[1] V. Bengin, and D. Budimir, "Integrated Waveguide Bandpass Filters Using Thick-Film Technology," IEEE, Microwave Review, June 2004.

[2] K. E. G. Pitt, Handbook of Thick Film Technology, Electrochemical Publications Ltd, 2005.

[3] M. Himdi, J. .P. Danial, and C. Terret, "Transmission Line Analysis of Aperture_coupled Microstrip Antenna," Electronics Letters, vol. 25, no. 18, , pp. 1229-1230, Jun. 1989.

[4] C. Ng, M. Chongcheawchaman, M. Aftanasar, I. Robertson, and J. Minalgiene, "X-band Microstrip Bandpass Filter using Photoimageable Thick-Film materials," 2002 IEEE MTT-S Int. Microwave Symp. Dig., vol. 3, pp.2209-2212, 2-7 June 2002

[5] T. C. Edwards, and M. B. Steer, Foundations of Interconnect and Microstrip Design, J. Wiley \& Sons, 2000.

[6] L. Tsai and C. Hsue, "Dual-Band Bandpass Filters Using Equal-Length Coupled-Serial-Shunted Lines and Z-Transform Technique," IEEE Trans. Microwave Theory \& Tech., vol. 52, no. 4, April 2004.

[7] K.J. Williams, A. Curley, M. Tunnicliffe, P. Barrett, P.J. Scott, and J. Ferguson "Thick Film Technology for Wireless Applications," IEEE EUROCON2001, vol. 1, pp. 178-183, 2001.

[8] P. Barnwell, and J. Wood, "Fabrication of Low Cost Microwave Circuits and Structures using An Advanced Thick Film Technology," 1998 IEMT/MC Symp., pp. 327-332, 15-17 April 1998. 